\title{
Personality predicts the propensity for social learning in a wild primate
}

Social learning can play a critical role in the reproduction and survival of social animals. Individual differences in the propensity for social learning are therefore likely to have important fitness consequences. We asked whether personality might underpin such individual variation in a wild population of chacma baboons (Papio ursinus). We used two field experiments in which individuals had the opportunity to learn how to solve a task from an experienced conspecific demonstrator: exploitation of a novel food and a hidden item of known food. We investigated whether the (1) time spent watching a demonstrator and (2) changes in task-solving behaviour after watching a demonstrator were related to personality. We found that both boldness and anxiety influenced individual performance in social learning. Specifically, bolder and more anxious animals were more likely to show a greater improvement in task solving after watching a demonstrator. In addition, there was also evidence that the acquisition of social information was not always correlated with its use. These findings present new insights into the costs and benefits of different personality types, and have important implications for the evolution of social learning. 
1 Alecia J. Carter ${ }^{1,2,}$, Harry H. Marshall ${ }^{2,3,4}$, Robert Heinsohn ${ }^{1}$ \& Guy Cowlishaw ${ }^{2}$

$2{ }^{1}$ The Fenner School of Environment and Society, The Australian National University,

3 Acton, Canberra, ACT, Australia

$4 \quad{ }^{2}$ The Institute of Zoology, Zoological Society of London, Regent's Park, London, UK

$5 \quad{ }^{3}$ Division of Ecology and Evolution, Department of Life Sciences, Imperial College

6 London, Silwood Park, Berkshire, UK

$7 \quad{ }^{4}$ Present address: Centre for Ecology and Conservation, College of Life and

8 Environmental Sciences, University of Exeter, Cornwall, TR10 9EZ, UK

9 "Author for correspondence: Alecia Carter, Large Animal Research Group, Department

10 of Zoology, University of Cambridge, United Kingdom T: +44 $1223769277 \mathrm{E}$ :

11 ac854@cam.ac.uk 


\section{INTRODUCTION}

Individuals can acquire information either through interacting with their environment directly—personal information—or through observing the behaviour of other individuals —social information (Dall et al. 2005). Personal information can be costly and time consuming to collect (Laland 2004). For social animals, social information provides an alternative to gathering costly personal information, potentially facilitating the acquisition of information about when, where and how to get food, where to travel and remain, whom to choose as a mate, and which predators to avoid (Giraldeau et al. 2002; Reader \& Biro 2010). Social learning-where an individual changes its behaviour after observing another-allows the rapid dissemination of novel information among group members, and has also been widely implicated in the formation of tradition and cultures within species (Whiten 2000; Castro \& Toro 2004). On the other hand, social learning can be conceived as a form of 'information parasitism' and can be maladaptive if information rapidly becomes outdated (Laland 2004). Nonetheless individual differences in innovation and social learning abilities remain largely overlooked (Thornton \& Lukas 2012). This is surprising, as an understanding of individual variation in social learning is likely to be necessary for predicting the formation and persistence of traditions and culture within groups, and the individual fitness advantages of personal versus social information use (Thornton \& Lukas 2012).

Animal personality refers to between-individual differences in behaviour that persist through time (Sih et al. 2004). Evidence for personality-related individual

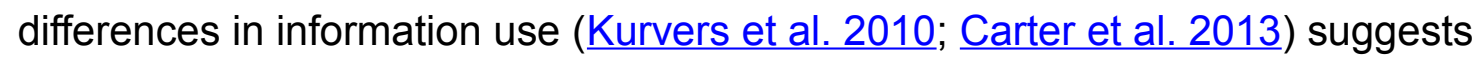
personality may also explain individual differences in social learning (Marchetti \& Drent 2000). This may occur at two different steps in the social learning process, when (1) a 
subset of individuals initially gathers personal information about a novel situation and (2) this subset - the 'demonstrators' - provide other individuals with the opportunity to then gather and use social information about the novel situation from them. In the first step, for individuals to exploit a novel food or solve a novel task they must interact with it for long enough to obtain sufficient personal information about it (Seferta et al. 2001;

Thornton \& Samson 2012). Individual variation in responses to novel stimuli are frequently used to differentiate individuals by two personality traits, namely boldness and exploration ( (Carter et al. 2012b). Those animals that are less bold, i.e. shyer, or less explorative of novelty, i.e. 'neophobic', individuals (Réale et al. 2007; Carter et al. 2012c) tend to be less willing to interact with a novel food or task and are thus less able to acquire the personal information necessary to solve the problem (but see Cole \& Quinn 2011). For example, neophobic myna (Acridotheres tristis) are less likely to solve innovation tasks than neophilic myna (see also Coleman et al. 2005; Toxopeus et al. 2005; Sol et al. 2011). In the second step, those animals that are less willing or able to gather personal information may be more likely to use social information. For instance, where individuals seek to exploit a novel food or solve a novel task, bolder individuals may do so directly because they are willing to gain personal information about it, and subsequently become demonstrators for shyer individuals who may be more likely to attend to, and act upon, social information (Kurvers et al. 2010).

We investigated the relationship between animal personality and social learning in wild chacma baboons (Papio ursinus). Baboons present an ideal system to explore such individual differences, since they are highly social, live in stable groups, and social learning has previously been documented among infants and juveniles (Whiten 2000). In addition, individual baboons in our study population vary consistently in two unrelated 
personality traits, boldness and anxiety, that are repeatable over a three-year period and for which all individuals in the study groups have been assayed up to six times (Carter et al. 2012c). We use the term 'boldness' to refer to an individual's response to a novel food, where bolder individuals spend more time inspecting a novel food; and 'anxiety' for an individual's response to a threatening stimulus, where more anxious individuals show a stronger fear response towards, and spend more time investigating, a threat (Carter et al. 2012c). Though threat responses can also be used to describe boldness, we have previously found no evidence that our threat presentation test measures boldness in this species (for further details, see Carter et al. 2012c).

To test our hypothesis that personality traits (boldness, anxiety) influence individual propensity for social learning, we performed two types of field experiments in which social learning to solve a task could occur: exploitation of a novel food (experiment NF) and a hidden food (HF). We investigated whether (Q1) propensity to solve the tasks using personal information alone, (Q2) time spent collecting social information (watching a demonstrator solve the tasks), and (Q3) social learning, i.e. changes in task-solving behaviour after exposure to a demonstrator, were related to the baboons' boldness or anxiety. Thus we addressed three questions with two experiments for two personality traits that could influence social learning.

We had several predictions in line with our questions. First, we predicted that bolder baboons would be more likely to 'solve' the hidden food task as bolder individuals are more willing to interact with a novel food (Carter et al. 2012a) (Prediction 1 for boldness: $\left.\mathrm{P} 1_{\mathrm{HF}} \mathrm{b}\right)$. We further predicted that there would be no relationship between anxiety and the propensity to solve the hidden food task as anxiety is unrelated to boldness in this species (Carter et al. 2012c) and anxious individuals are otherwise no 
more likely to interact with a novel food than calm individuals ( $\left.\mathrm{P} 1_{\mathrm{HF}} \mathrm{a}\right)$. Second, regarding whether an individual would collect social information, we predicted that more anxious baboons would spend more time watching the demonstrator than calmer baboons, in both the novel food and hidden food tasks, as anxious baboons attend to a threatening stimulus for longer than calm baboons (Carter et al. 2012C) (P2 $\left.2_{\mathrm{NF}, \mathrm{HF}} \mathrm{a}\right)$. We further predicted that shyer baboons would spend more time watching a demonstrator than bolder baboons in both tasks as shy geese attend to social information more than bold geese (Kurvers et al. 2010) (P2 $\left.2_{\mathrm{NF}, \mathrm{HF}} \mathrm{b}\right)$. Finally, regarding whether individuals differed in their propensity for social learning in the novel food and hidden food tasks, we predicted that more anxious individuals $\left(\mathrm{P} 3_{\mathrm{NF}, \mathrm{HF}} \mathrm{a}\right)$ would show greater social learning than calmer baboons, as anxious individuals were predicted to attend to social information more than calm baboons (P2 ${ }_{\mathrm{NF}, \mathrm{HF}} \mathrm{a}$ above). Similarly, we predicted that shyer individuals (P3 $\left.3_{\mathrm{NF}, \mathrm{HF}} \mathrm{b}\right)$ would show greater social learning than bold baboons in both tasks, as shy individuals were predicted to attend to social information more than bold baboons ( $\mathrm{P} 2_{\mathrm{NF}, \mathrm{HF}} \mathrm{b}$ above).

\section{MATERIALS AND METHOD}

\section{Study area and species}

We studied the chacma baboons over three years, from May to November 2009, May to October 2010 and June to September 2011 at Tsaobis Leopard Park, Namibia (15 $45^{\prime} E, 22 \square 23$ 'S). Two groups of baboons ( $n=44,31$ in 2009) have been habituated to the presence of observers at close range and are individually recognisable. We collected data annually from 57 adult, subadult and juvenile baboons (we did not test individuals who were dependent young in 2009) over the 2009 to 2011 period. Individuals were 
assigned to age classes (juvenile/adult) on the basis of canine eruption in males and menarche in females (after which they were considered adult). Age (years) was estimated from a combination of known birth dates and dental patterns of tooth eruption and wear (Huchard et al. 2009). Individual ranks were assessed through dominance interactions that were recorded during focal observations and ad libitum using Matman 1.1.4 (Noldus Information Technology 2003). Hierarchies in both troops were strongly linear in all years (in 2010, for example, Landau's corrected linearity index: $h^{\prime}{ }_{\text {larger troop }}=$ $0.71, h_{\text {smaller troop }}^{\prime}=0.82, p<0.001$ in both). Rank was expressed as a relative rank (which controls for group size), calculated from absolute ranks for each baboon using the formula 1-[(1-r)/(1-n)] where $r$ is the individual's absolute rank and $n$ is the group size of the individual.

\section{Personality assessments}

Boldness and anxiety were assessed using an experimental approach in 2009, 2010 and 2011 (for further details, see Carter et al. 2012a; Carter et al. 2012c).

Boldness was assessed by scoring responses to a novel food, while anxiety was assessed by scoring responses to a venomous snake (Carter et al. 2012c). In all cases, individuals were presented with a stimulus when they were alone and moving between food patches. The stimuli were presented on the edges of game trails and paths regularly used by the baboons. All experiments were filmed to facilitate data extraction (Panasonic SDR-SW20, Kadoma Osaka, Japan; see movie files in Carter et al. 2012c).

Stimuli for the boldness experiments consisted of novel food items which included hard-boiled eggs with the shell on or removed, or a small egg-shaped bread roll, all of 
which were dyed red or green (Moir's food dye), in 2009; semi-dried eighths of apple or pear, dyed red, in 2010; and eighths of an orange or equivalent-sized pieces of butternut squash in 2011. Any naïve individual that saw another individual interacting with a novel food was presented with a different novel food when they were tested. We recorded the latency in seconds (s) to approach the food item on detection (if the food item was not approached, the individual was given the maximum value of $150 \mathrm{~s}$; individuals that did not detect the item were subsequently re-tested), the time spent inspecting the food item ( $s$; the time between approaching the food item and the end of the experiment, either leaving or eating the item), and the time spent handling the food item (s; the time spent touching the food item). Fifty-eight, fifty-four and fifty baboons received novel food presentations in 2009, 2010 and 2011, respectively (mean and median number of presentations $=2.8,3.0$; range $=1-3$ presentations $)$.

The stimulus for the anxiety experiments was a taxidermic puff adder Bitis arietans. We recorded as binary responses whether an individual approached, vocalised, performed a self-directed behaviour, bared its teeth, flagged its tail, stopped and backed away (see Carter et al. 2012c for details). If more than one baboon saw the stimulus during a given presentation, we extracted data for both of the individuals if the second individual did not respond to the reaction of the first individual (and in one case third individual). A baboon was recorded as responding to another individual if it looked in the direction of and/or approached the individual who initially saw the stimulus. In total, we completed 153 snake presentations to 57 baboons over 2009 to 2011 (mean and median number of presentations $=2.7,3.0$; range $=1-6$ presentations $)$.

$151=0.26,0.34$, respectively) ( Carter et al. 2012c), we used a composite score for each trait 
152 from trait values collected over three years (see Carter et al. 2012c). Boldness and

153 anxiety are independent in this species, thus a bolder baboon is equally likely to be as

154 anxious as a shyer baboon (Carter et al. 2012c).

\section{Social learning experiments}

156 From 2009 to 2011 , we performed two types of experiment designed to assess

157 differences in social learning in baboons: a novel food experiment and a hidden food experiment. In both cases, we attempted to adopt a 'test - learn (via a demonstrator) re-test' experimental design. The experiments were run concurrently to limit order

160 effects. All experiments were filmed to facilitate data extraction, and the baboons' 161 identities and activities were dictated to the camera (Panasonic SDR-SW20, Kadoma

162 Osaka, Japan; see Supplemental Video 1). The study population in 2010 comprised 21 163 male and 34 female baboons who were on average (mean) 8.2 years old (range: 4-21), 164 had a relative rank of $0.5(0-1)$, inspected the novel food on average for $20 \mathrm{~s}(0-120)$ 165 with an mean boldness score of $0.46(-3.90-1.47)$ (note that low values represent 166 individuals that inspected the food for longer, such that high scores indicate shyer 167 animals) and an average anxiety score of $-0.02(-0.22-0.53)$ (where high scores indicate 168 more anxious animals).

170 The 'test' stage of the novel food experiments was also our assay for boldness (see 171 above). Thus, solitary individuals were presented with a novel food while moving 
172 between natural food patches. In the majority of cases, the test subjects only briefly

173 inspected the food and failed to eat it (Carter et al. 2012a). In the 'learn' stage, the same

174 individuals were subsequently given the opportunity to watch an experienced

175 demonstrator with the same novel food. The demonstrator was an individual who had

176 encountered the novel food at least once before, and had consumed at least part of it on

177 the last encounter. We achieved this by presenting a novel food item to small subgroups

178 (two-five baboons within $5 \mathrm{~m}$ of one another) that included a randomly-chosen

179 demonstrator. We recorded the identities of any additional baboons that came within $5 \mathrm{~m}$

180 of the demonstrator (and could therefore watch the demonstrator) and the duration that

181 the (potential) observers watched the demonstrator (seconds). Watching the

182 demonstrator was defined as an individual directing its gaze towards the demonstrator.

183 The difference in dominance ranks between the observers and the demonstrator was

184 also recorded.

In the final 're-test' stage, we subsequently presented the non-demonstrators with

186 the novel food again, under the same conditions as in the initial test stage (i.e. while each was solitary and moving between food patches). All observers were tested after their 'learn' trial, but could also have been observers in others' 'learn' trials subsequently

189 (as we could not control subgroup formation). If the non-demonstrators had learned from watching the demonstrator, we expected their responses to the novel food to differ from

191 that previously observed. Specifically, we expected shy and anxious individuals'

192 handling time, which positively correlates with the probability of eating the novel food

193 (Carter et al. 2012a), to increase on the 're-test' trial compared to the initial trial, while 194 bold and calm individuals' behaviour would not change (Table 1). 
As a control, we also re-tested a subset of baboons that did not have the

opportunity to watch a demonstrator and therefore could only change their responses to the 'novel' food through further personal experience. The 'social learning of novel food' experiment was carried out on 16 subjects in 2010; the control trials were carried out on 23 subjects between 2009 and 2011. The demonstrators for this experiment comprised 6 male and 7 female baboons who were on average 6.8 years old, had a relative rank of 0.58 , inspected the novel food on the first presentation on average for $51 \mathrm{~s}$ with an average boldness of -1.17 and an average anxiety of 0.05 . The focal subjects for this experiment comprised 13 male and 19 female baboons who were on average 7.6 years old, had a relative rank of 0.48 , inspected the novel food on the first presentation on average for $19 \mathrm{~s}$ with an average boldness of 0.15 and an average anxiety of -0.01 .

\section{Hidden food experiments}

In the hidden food experiment, the subjects were presented with a hidden-food task whilst they were moving with other baboons between food patches. The hidden-food task consisted of an empty, opaque $1 \mathrm{~L} \mathrm{UHT} \mathrm{milk} \mathrm{tetrapack} \mathrm{that} \mathrm{had} \mathrm{been} \mathrm{rinsed}$ thoroughly and dried. One corner of the top of the tetrapack had been cut off to let the milk out, and the other corner unfolded so that the top, short end of the tetrapack was no longer flat. Fifteen to twenty dried maize kernels - a highly preferred food for the baboons - were placed in the tetrapack. Since the tetrapack was opaque, the baboons had to interact closely with it to find the food inside. We doubt that the baboons could smell the maize kernels, but if they could they would have had to interact intimately with the stimulus to do so. Individuals could discover the food by tipping the tetrapack upside down, or ripping the tetrapack open. 
219 the baboons that came within $5 \mathrm{~m}$ of the stimulus when there was no 'demonstrator', and thus had an opportunity to obtain personal information about the stimulus; the identities of the baboons that interacted with the stimulus, defined as approaching to smell, touch or handle the tetrapack, and whether they solved the task, defined as eating any food from the tetrapack; the identities of the baboons that came within $5 \mathrm{~m}$ of a demonstrator, i.e. an individual interacting with the stimulus; and whether they watched the demonstrator, defined as looking in the direction of the demonstrator while stationary, or not, which was defined as not looking in the direction of the demonstrator, or glancing at the demonstrator but not stopping to watch, while at this proximity. The difference in dominance rank between each demonstrator and observer was also recorded.

We encountered two unanticipated difficulties with this series of experiments.

First, subordinates occasionally moved away carrying the stimulus when they were approached by dominants. In these cases, the experimenter followed the baboon with the stimulus but did not record the 'watching' durations of the observers, as these were unlikely to be accurate. Second, because individuals moved with the task through the troop as it was foraging, it was impossible to present the task to particular individuals in a particular order. Individuals were thus sometimes able to experience a 'learn' trial before a 'test' trial, and we were unable to systematically achieve the more controlled test - learn - re-test experiments of the novel food task. Further, we were unable to control the number of times an individual was tested with or without a demonstrator. However, this situation is more likely to reflect the natural opportunities for social 241 learning than our more controlled test — learn — re-test paradigm, and we have 
242 therefore included this series of experiments in our analyses. In this case, for each

243 individual, we recorded three possible trial presentation numbers that reflect the different

244 types of information that they could acquire: the cumulative presentation number

245 (cumulative total social and personal information), the number of presentations with a

246 demonstrator (cumulative social information) and the number of presentations without a

247 demonstrator (cumulative personal information).

Because we retrieved a task after it had been solved, no baboons could have had

a 'learn' presentation (with a demonstrator) and a 're-test' presentation (without a

demonstrator) in the same trial. That is, no individuals could watch a demonstrator and

then subsequently interact with an unsolved task, though some individuals did interact

with parts of the task that were ripped off and dropped by the demonstrator before the

experimenter could retrieve them. Individual baboons experienced the task with a

demonstrator a median of 3 times (inter-quartile range $(I Q R)=2-5$, range $=1-11$ times)

and without a demonstrator a median of 2 times $(I Q R=1-3$, range $=1-9$ times $)$, and in

total (either with a demonstrator or without) a median of 5 times $(I Q R=1-3$, range $=1$ -

16 times) with a mean interval of $3.30 \pm 0.47$ days (range $=0-52$ days) between trials.

We performed a total of 243 baboon-trials to 45 baboons with 18 demonstrators who

were demonstrators for a median of 5.5 individuals $(I Q R=4-15.5$, range $=1-25$

individuals). The demonstrators for this experiment comprised 11 male and 6 females

261 (and one infant) who were on average 6 years old, had a relative rank of 0.64 , with an

265 anxiety of -0.03. 
We confirm that we have adhered to the Guidelines for the Use of Animal Behaviour for Research and Teaching (Anim. Behav. 2003. 65, 249-255). Our experimental protocols were assessed and approved by the Ethics Committee of the Zoological Society of London (BPE/052). Our study was approved by the Ministry of Environment and Tourism in Namibia (Research/Collecting Permits 1379/2009, 1486/2010, and 1486/2011).

\section{Statistical analyses}

\section{General modelling approach}

All data were analysed using (generalised) linear mixed effects models (LMEs or GLMMs), fitted in the R environment ( $\underline{R}$ Development Core Team 2011). Unless otherwise specified below, all models started with all fixed and random effects, and fixed effects were sequentially dropped (from least significant terms first) until a minimal model was obtained that included only those effects which explained a significant amount of the variation in the model. Each dropped term was added to the minimal (final) model to check that it remained non-significant. Significance of terms was determined by $p$-values (all effects with a $p$-value $\leq 0.05$ were retained in the models). Full models included the following fixed effects: boldness, anxiety, sex (factor: male or female), age class (factor: juvenile or adult), and rank (continuous variable: standardised between 0 and 1 within each troop). We also included the rank difference between the test subject and the demonstrator in those models investigating whether or not the subject watched the demonstrator (continuous variable; see models 2 described below).

Finally, we included presentation number as a fixed effect in all hidden food task models 
as individuals were exposed to the stimuli on multiple occasions in this experiment. In all models, we included test subject identity and troop identity (to control for repeated measures) as random effects. The latter was further crossed with demonstrator identity in those models investigating whether or not the test subjects watched a demonstrator (models 2). All Gaussian models' residuals were normally distributed, and there were no covariances $>0.40$ between fixed effects.

\section{Novel food experiments}

We did not analyse which individuals initially solved the novel food task (Q1), since the processing and consumption of the novel food was the basis of our boldness assay. We thus first investigated whether individuals of different personality types spent longer watching a demonstrator (Q2, predictions $2 \mathrm{a}$ and b, i.e. P2 ${ }_{\mathrm{NF}} \mathrm{a}$ and $\left.\mathrm{b}\right)$. Using data obtained from the novel food experiments when a demonstrator was interacting with the food ( $n=54$ baboon-trials with 36 baboons observing 14 demonstrators), we analysed the time the baboons spent watching the demonstrator (s) with a Poisson error structure (model 2, novel food: $\mathrm{m} 2 \mathrm{NF}_{\mathrm{NF}}$.

Next (Q3), we investigated whether individuals of different personality types were more or less likely to change their behaviour, i.e. learn, after being exposed to social information via a demonstrator (predictions $3 a$ and b, i.e. P3 $3_{\mathrm{NF}} \mathrm{a}$ and b). We investigated whether the baboons were more likely to increase their handling time of a novel food after watching a demonstrator, and whether this depended on boldness or anxiety, by investigating whether there was a three-way interaction between the personality trait, the presentation ('test' or 're-test'), and the treatment (control or treatment) (models $3_{\mathrm{NF}}$; 
309

310

311

312

$313\left(\mathrm{~m} 3_{\mathrm{NF}} \mathrm{a}\right.$ and $\left.\mathrm{b}\right)$.

\section{Hidden food experiments}

315 First (Q1), we investigated whether personality predicted whether individuals found food in the hidden food experiment (i.e. solved the task; see predictions $\mathrm{P} 1_{\mathrm{HF}} \mathrm{a}$ and b). We analysed whether an individual managed to solve the hidden-food task if it had an opportunity to do so (i.e. obtain food from it, binary $1 / 0 ; n=80$ occasions when a baboon interacted with the tetrapack, involving 33 different baboons) using a GLMM with a binomial error structure (model 1, hidden food experiment: $\mathrm{m} 1_{\mathrm{HF}}$ ). As previously mentioned, few individuals had the opportunity to interact with the task without previously having seen a demonstrator interacting with the task; consequently, this analysis cannot distinguish between task-solving due to 'innovation' or social learning 324 (but see Results).

Next, we investigated whether individuals of different personality types were more likely to watch a demonstrator (Q2, predictions $\mathrm{P} 2_{\mathrm{HF}} \mathrm{a}$ and b) using data obtained from

327 the hidden-food experiments with a demonstrator. We analysed whether individuals watched the demonstrator or not ( $n=163$ baboon-trials with 41 baboons observing 14 demonstrators), using a binomial error structure (model $2_{\mathrm{HF}}$ ). 
Finally, we investigated whether the baboons were more likely to increase their response to the hidden-food stimulus after watching a demonstrator, and whether this depended on boldness or anxiety by investigating whether there was an interaction between either of these traits and the presentation number (Q3, predictions $\mathrm{P} 3_{\mathrm{HF}} \mathrm{a}$ and b). In this case, we categorised the subjects' responses on a five-point scale, coded 0-4 in order of increasing response to the stimulus, as: ignore, the individual walked within 5 $\mathrm{m}$ of the stimulus without showing a response; look, the individual walked or stopped within $5 \mathrm{~m}$ of the stimulus and look towards it; smell, the individual approached the stimulus and bend to smell it; handle, the individual approached the stimulus and picked it up; or eat, the individual obtained food from the stimulus. As the models' residuals were normally distributed when analysed with a Gaussian error, we used linear mixed effects models to model the data (models $3_{\mathrm{HF}}$ ) rather than generalised linear mixed models for ordinal data. Due to the design of the hidden food task, subjects varied in the number of presentations they had experienced. To allow for this variation, we ran three models with different predictors: the cumulative number of all presentations (total exposure), the cumulative number of 'test' presentations without a demonstrator, and the cumulative number of 'learn' presentations with a demonstrator. We used backward model selection on all three models, each including one of the different cumulative presentation numbers (total, personal and social information presentations) and then compared with a log likelihood ratio test which of the three minimal models best explained the data, which included 80 baboon-trials to 33 individuals. 
352 The minimal models for all analyses described below are provided in Table 1, and a

353 summary of our findings with regard to our predictions is provided in Table 2 . In addition

354 to the results we present below regarding personality, it is notable that age class had an

355 effect on many of the variables that we investigated (Table 1). In all cases, there was a

356 positive effect of being juvenile on our measured variables: juveniles solved the hidden

357 task more often $\left(\mathrm{m}_{1 \mathrm{HF}}\right)$, spent longer watching a demonstrator $\left(\mathrm{m} 2_{\mathrm{NF}}\right)$, and showed

358 evidence for greater social learning than adults: juveniles handled the novel food for

359 longer after watching a demonstrator $\left(\mathrm{m} 3_{\mathrm{NF}} \mathrm{a}\right)$, and showed greater responses to (and

360 improvement in, see Supplemental File 1) the hidden food task ( $\left.\mathrm{m} 3_{\mathrm{HF}}\right)$. Few other

361 variables had such a consistent effect on our measurements (Table 1).

362 Novel food experiments

363 We assessed individual variation in the time spent observing demonstrators manipulate

364 the novel food (Q2). We found no evidence that shyer baboons were more attentive

365 observers, contrary to $\mathrm{P} 2_{\mathrm{NF}} \mathrm{b}$. Further, in contrast to $\mathrm{P} 2_{\mathrm{NF}} \mathrm{a}$, calmer baboons spent more

366 time watching demonstrators interacting with a novel food, after accounting for the

367 subject's age and rank ( $\mathrm{m} 2_{\mathrm{NF}} \beta \pm$ s.e. $=-2.79 \pm 0.51, t=-2.01$, Fig. 1$)$.

Next, we assessed the evidence for social learning, and individual variation in social learning arising from personality differences. In the case of novel foods, we achieved this by analysing the food handling time in the 're-test' conditions both for those baboons that either had no exposure to a demonstrator after the initial test (the control group) and for those that did (the experimental group). In both the models for anxiety (a) and boldness (b) (models $3_{\mathrm{NF}} \mathrm{a}$ and b), the three-way interaction was 
374 significant $\left(3_{\mathrm{NF}} \mathrm{a}: \beta \pm\right.$ s.e. $=7.66 \pm 3.04, \mathrm{z}=2.52, p=0.01 ; 3_{\mathrm{NF}} \mathrm{b}: \beta \pm$ s.e. $=-0.68 \pm 0.08, \mathrm{z}$

$375=-8.67, p<0.001)$. Overall, in the control groups, individuals spent less time handling

376 the novel food when re-tested, and both bolder and more anxious individuals showed a

377 greater decline in handling time when re-tested (Table 1; Fig. 2a, c). In the treatment

378 groups, individuals showed the opposite response-handling times tended to increase

379 when re-tested—and bolder and more anxious individuals showed higher handling times

380 than shyer and calmer individuals, respectively (models $3_{\mathrm{NF}}$ a and b, Table 1; Fig 2b, d).

381 Although calmer individuals also showed higher handling times in the second

382 presentation after watching a demonstrator, this reflected a non-significant 1.4-fold

383 increase in handling time in comparison to the 2.7 -fold increase in more anxious

384 individuals. Thus, bolder and more anxious animals showed social learning after

385 exposure to a demonstrator (contrary to $\mathrm{P} 3_{\mathrm{NF}} \mathrm{b}$, but in support of $\mathrm{P} 3_{\mathrm{NF}} \mathrm{a}$, respectively).

Hidden food experiments

In our assessment of individual propensity for task-solving (Q1), we found that bolder baboons were more likely to obtain food from the hidden food experiment $(\beta \pm$ s.e. $=$ $-0.85 \pm 0.32, z=-2.63, p=0.008$ ), after accounting for differences in age (model 1 , Table 1; Fig. 3), in support of P1 $\mathrm{HF}$ b. Anxiety, however, had no effect on task-solving, in support of $\mathrm{P} 1_{\mathrm{HF}}$ (model $1: \beta \pm$ s.e. $=-0.28 \pm 0.72, z=-0.40, p=0.69$ ).

We then assessed individual variation in the probability of observing the demonstrators (Q2). We found no evidence that shyer or more anxious baboons were more likely to watch an individual manipulate the hidden food task, contrary to $\mathrm{P}{ }_{\mathrm{HF}} \mathrm{b}$ and 395 a respectively (Table $1, \mathrm{~m} 2 \mathrm{HF})$. 
Finally, we assessed the evidence for social learning by analysing the intensity of

the response to the stimulus in relation to the number of prior total, social or personal presentations. We found no evidence for an interaction between any of the presentation numbers and either personality trait; in all cases the minimal model was the same (model $3_{\mathrm{HF}}$ ). Thus, while bolder animals continued to be better at solving the hidden food task (as observed in $\mathrm{m} 1$ ), we found no evidence that either shyer or more anxious individuals showed improved responses with more prior experience, contrary to predictions $3_{\mathrm{HF}} \mathrm{b}$ and $3_{\mathrm{HF}} \mathrm{a}$ respectively.

\section{DISCUSSION}

Our results strongly suggest that personality predicts social learning propensity in wild baboons. In a field experiment in which individuals had the opportunity to learn about a novel food from an experienced demonstrator, both bolder and more anxious individuals increased their handling time of the novel food if they had had an opportunity for social learning. Below we address those predictions that were not supported before focussing on three important issues emerging from our study that have implications not only for understanding social learning in the wild (van de Waal \& Bshary 2011), but also for individual differences in cognitive abilities (Thornton \& Lukas 2012) and the formation of traditions and culture in animal groups (Whiten 2000).

Contrary to expectation, we found no effect of boldness on time spent watching a demonstrator. These results differ from those of Kurvers et al. (2010) which suggested that shy individuals used social information more than bold individuals, but this discrepancy may be explained by differences between studies in the familiarity of the 
study subjects with the experimental challenge (see below). However, our finding that bold individuals were more likely to solve the hidden food task confirms previous research showing an increase in task-solving success when individuals are less neophobic (Seferta et al. 2001; Webster \& Lefebvre 2001). Our failure to find a comparable improvement in task solving after watching a demonstrator for the hidden food experiment is unexpected, but may reflect the fact that the task contained a highly preferred food, and individuals may be limited by their rank in their access to the task. That is, individuals may have acquired the social information necessary to solve the task, but were unable or unwilling to access the task due to their lower rank. Though we do not have the resolution of data to test this explicitly, we discuss this possibility further below.

Three important issues emerged from our study. First, an individual's ability or interest in collecting social information does not necessarily correlate with its ability or interest in using social information. This observation arises from our findings that bolder and more anxious individuals showed a greater improvement than shyer and calmer individuals in their response to a novel food after watching a demonstrator, despite the fact that there was no effect of boldness on the time spent watching a demonstrator, and calmer individuals were more attentive than more anxious individuals. Three possible explanations could be considered for these results: that (i) a mechanism other than watching a demonstrator is responsible for social learning in baboons, (ii) baboons need little time to acquire sufficient social information to subsequently change their behaviour, or (iii) as mentioned above, the acquisition of social information is not always correlated with its use. The first explanation is unlikely, since the task is a visual one and baboons 
are strongly visual animals. It therefore seems probable that the second or third explanations are most likely, and these need not be mutually exclusive.

Social learning mechanisms such as stimulus enhancement, where the behaviour or presence of a demonstrator attracts the attention of a naïve individual (Brown \& Laland 2003), may quickly result in a behavioural change in naïve individuals without the detailed and lengthy observation assumed necessary in processes such as imitation. As such, the baboons could learn that an object is worth investigating in more detail in the future in the time it takes to identify the object that a demonstrator is manipulating, as may be the case in our study. Further, it may be that bold individuals show greater social learning not necessarily because they are 'better' at collecting or processing social information, but because they are generally more willing to interact with a novel task. This, coupled with the rapid social learning through stimulus enhancement we posit to be possible in this study, may result in bolder individuals showing greater social learning. In support of this interpretation, in the novel food control trials, bold baboons decreased their handling time of the novel food but this handling time still exceeded that of shy individuals on their first presentations. This may also explain why our findings differ to those of Kurvers et al. (2010), as the authors of that study habituated the study subjects to the experimental arena prior to testing. In both cases (our study, and Kurvers et al.'s study), individual variation in response to novel tasks is related to personality, but not necessarily an individual's ability to collect and process social information. The fact that some individuals may be too shy or anxious to interact with a novel task, despite having the information or ability to do so, may have wide implications. For example, an individual's cognitive performance may be systematically underestimated if it is unwilling, but still able, to solve a task, which may hinder studies interested in the fitness 
advantages and evolution of cognitive performance. Future studies could assess this effect experimentally by testing study animals of different boldness with varying degrees of habituation to a test apparatus. Our interpretation would lead to the prediction that bolder individuals should outperform shyer individuals under low habituation regimes, but that performance may be more equal when the task is no longer threatening.

Second, the identity of demonstrators may be important. In both our experiments, many of our initial demonstrators were bold. In the novel food experiment, this was inevitable, as our definition of boldness precluded individuals that did not eat the novel food. However, it also transpired that those individuals that solved the hidden food task were more likely to be bold, though we note that the boldness and anxiety of the demonstrators was, overall, not particularly different to the boldness and anxiety of the population (see summary statistics reported in the methods Methods for these groups). As these experiments were not designed to address this particular question and thus the sample of demonstrators is limited, we have not analysed these data here. However, previous research has suggested that the characteristics of a demonstrator may affect whether an observer gathers or uses social information from them or not (termed 'directed social learning': Coussi-Korbel \& Fragaszy 1995; Laland 2004). For instance, wild vervet monkeys (Chlorocebus aethiops) are more likely to attend to the social information provided by the philopatric sex (females) (van de Waal et al. 2010). It remains to be established whether the personality of the demonstrator might be important, but it seems possible that it may play a role. For example, slow explorers are more thorough at exploring novel environments (Careau et al. 2009) and may therefore be better informed about the location of resources. Although it is difficult to anticipate how demonstrator boldness might affect audience attentiveness and social learning in 
baboons, we can consider its correlates. Specifically, while sex is unrelated to boldness in our population, there is an effect of age: juvenile baboons are bolder than adults (unpublished data). Since this led to a strong representation of juvenile animals among the demonstrators (10 juvenile, 4 adult demonstrators), and adults may attend less closely to juveniles (only vertical transmission of social information has been documented in this species: (Camberfort 1981)), it is possible that the adults in our sample might have shown lower levels of attentiveness and social learning than would have been observed with adult-only demonstrators.

Finally, an important aspect of our study that we did not anticipate was the difficulty of testing certain individuals in the presence of a demonstrator. While we endeavoured to provide all individuals with the opportunity to watch a demonstrator for both tasks, it was not possible to find a situation in which certain individuals were foraging in sufficiently close proximity to potential demonstrators for this to occur (Sih \& Del Giudice 2012). In great tits (Parus major), slow exploring birds showed higher attraction to unknown conspecifics than fast explorers (Carere et al. 2001) suggesting that personality may influence sociability and thus opportunities to learn from others in some species. Further, in guppies (Poecilia reticulata), an individual's social network is related to its personality: individuals associated assortatively according to behavioural type, and shy fish had more and stronger network connections than bold fish ( 2009). An individual's social network is thus likely to affect which individuals can supply social information, and thus the flow of information through a group (Voelkl \& Noë 2008; Sih et al. 2009; Claidiére et al. 2013). If similar personality-related patterning among the social networks of baboons occurs, it may be impossible for some individuals to obtain social information about a novel food or task as they may have only weak associations 
513 with demonstrators. Together with the preceding points, it thus appears possible that

514 personality could affect social learning, and hence the formation of culture in animal

515 groups, through a combination of mechanisms. These include not only the differential

516 collection of social information, as we have shown in this study, but also the differential

517 application of social information, directed social learning, and assortative social bonds. 
519 We thank Baboon Teams 2009, 2010 and 2011 for putting up with AJC standing around

520 and swearing at baboons a lot, especially Claudia Sick who performed some of the

521 novel food control presentations in 2009, and Drandrew Bateman, Corina Logan and

522 Dieter Lukas for commenting on an earlier version of the manuscript. We are grateful to

523 the Ministry of Lands and Resettlement for permission to work at Tsaobis Leopard Park,

524 the Gobabeb Training and Research Centre for affiliation, and the Ministry of

525 Environment and Tourism for research permission in Namibia. We are also grateful to

526 the Snyman and Wittreich families for permission to work on neighbouring farms. This

527 paper is a publication of the ZSL Institute of Zoology's Tsaobis Baboon Project. 
530

531

532

533

534

535

536

537

538

539

540

541

542

543

544

545

546

547

548

549

550

551

552

553

554

555

556

557

558

559

560

561

562

563

564

565

566

567

568

569

570

571

572

573

574

575

576

577

578

Brown, C. \& Laland, K. N. 2003. Social learning in fishes: a review. Fish and Fisheries, 4, 280288.

Camberfort, J. P. 1981. A comparative study of culturally transmitted patterns of feeding habits in the chacma baboon Papio ursinus and the vervet monkey Cercopithecus aethiops. Folia Primatologica, 36, 243-263.

Careau, V., Bininda-Emonds, O. R. P., Thomas, D. W., Réale, D. \& Humphries, M. M. 2009. Exploration strategies map along fast-slow metabolic and life-history continua in muroid rodents. Functional Ecology, 23, 150-156.

Carere, C., Welink, D., Drent, P. J., Koolhaas, J. M. \& Groothuis, T. G. G. 2001. Effect of social defeat in a territorial bird (Parus major) selected for different coping styles. Physiology \& Behavior, 73, 427-433.

Carter, A., Marshall, H., Heinsohn, R. \& Cowlishaw, G. 2012a. Evaluating animal personalities: do observer assessments and experimental tests measure the same thing? Behavioral Ecology and Sociobiology, 66, 153-160.

Carter, A. J., Feeney, W. E., Marshall, H. H., Cowlishaw, G. \& Heinsohn, R. 2012b. Animal personality: what are behavioural ecologists measuring? Biological Reviews, 88, 465-475. Carter, A. J., Marshall, H. H., Heinsohn, R. \& Cowlishaw, G. 2012c. How not to measure boldness: novel object and antipredator responses are not the same in wild baboons. Animal Behaviour, 84, 603-609.

Carter, A. J., Marshall, H. H., Heinsohn, R. \& Cowlishaw, G. 2013. Personality predicts decision making only when information is unreliable. Animal Behaviour, 86, 633-639.

Castro, L. \& Toro, M. A. 2004. The evolution of culture: From primate social learning to human culture. Proceedings of the National Academy of Sciences of the United States of America, 101, 10235-10240.

Claidiére, N., Messer, E. J. E., Hoppitt, W. \& Whiten, A. 2013. Diffusion Dynamics of Socially Learned Foraging Techniques in Squirrel Monkeys. Current Biology, 23, 1-5.

Cole, E. F. \& Quinn, J. L. 2011. Personality and problem-solving performance explain competitive ability in the wild. Proceedings of the Royal Society B: Biological Sciences, 279, 1168-1175.

Coleman, K., Tully, L. A. \& McMillan, J. A. 2005. Temperament Correlates With Training Success in Adult Rhesus Macaques. American Journal of Primatology, 65, 63-71.

Coussi-Korbel, S. \& Fragaszy, D. M. 1995. On the relation between social dynamics and social learning. Animal Behaviour, 50, 1441-1453.

Croft, D. P., Krause, J., Darden, S. K., Ramnarine, I. W., Faria, J. J. \& James, R. 2009. Behavioural trait assortment in a social network: patterns and implications. Behavioral Ecology and Sociobiology, 63, 1495-1503.

Dall, S. R. X., Giraldeau, L.-A., Olsson, O., McNamara, J. M. \& Stephens, D. W. 2005. Information and its use by animals in evolutionary ecology. Trends in Ecology \&amp; Evolution, 20, 187-193.

Giraldeau, L. A., Valone, T. J. \& Templeton, J. J. 2002. Potential disadvantages of using socially acquired information. Philosophical Transactions of the Royal Society B: Biological Sciences, 357.

Huchard, E., Courtiol, A., Benavides, J. A., Knapp, L. A., Raymond, M. \& Cowlishaw, G. 2009. Can fertility signals lead to quality signals? Insights from the evolution of primate sexual swellings. Proceedings of the Royal Society B-Biological Sciences, 276, 1889-1897.

Kurvers, R. H. J. M., van Oers, K., Nolet, B. A., Jonker, R. M., van Wieren, S. E., Prins, H. H. T. \& Ydenberg, R. C. 2010. Personality predicts the use of social information. Ecology Letters, 13, 829-837.

Laland, K. N. 2004. Social learning strategies. Learning \& Behavior, 32, 4-14. 
Marchetti, C. \& Drent, P. J. 2000. Individual differences in the use of social information in foraging by captive great tits. Animal Behaviour, 60, 131-140.

R Development Core Team. 2011. R: A language and environment for statistical computing. Vienna, Austra: R Foundation for Statistical Computing.

Reader, S. M. \& Biro, D. 2010. Experimental identification of social learning in wild animals. Learning \& Behavior, 38, 265-283.

Réale, D., Reader, S. M., Sol, D., McDougall, P. T. \& Dingemanse, N. J. 2007. Integrating animal temperament within ecology and evolution. Biological Reviews, 82, 291-318.

Seferta, A., Guay, P.-J., Marzinotto, E. \& Lefebvre, L. 2001. Learning Differences between Feral Pigeons and Zenaida Doves: The Role of Neophobia and Human Proximity. Ethology, 107, 281-293.

Sih, A., Bell, A. \& Johnson, J. C. 2004. Behavioral syndromes: an ecological and evolutionary overview. Trends in Ecology \& Evolution, 19, 372-378.

Sih, A. \& Del Giudice, M. 2012. Linking behavioural syndromes and cognition: a behavioural ecology perspective. Philosophical Transactions of the Royal Society B: Biological Sciences, 367, 2762-2772.

Sih, A., Hanser, S. F. \& McHugh, K. A. 2009. Social network theory: new insights and issues for behavioral ecologists. Behavioral Ecology and Sociobiology, 63, 975-988.

Sol, D., Griffin, A. S., Bartomeus, I. \& Boyce, H. 2011. Exploring or Avoiding Novel Food Resources? The Novelty Conflict in an Invasive Bird. PLOS ONE, 6.

Thornton, A. \& Lukas, D. 2012. Individual variation in cognitive performance: developmental and evolutionary perspectives. Philosophical Transactions of the Royal Society B-Biological Sciences, 367, 2773-2783.

Thornton, A. \& Samson, J. 2012. Innovative problem solving in wild meerkats. Animal Behaviour, 83, 1459-1468.

Toxopeus, I. B., Sterck, E. H. M., van Hoof, J. A. R. A. M., Spruijt, B. M. \& Heeren, T. J. 2005. Effects of trait anxiety on performance of socially housed monkeys in a learning test. Behaviour, 142.

van de Waal, E. \& Bshary, R. 2011. Social-learning abilities of wild vervet monkeys in a twostep task artifical fruit experiment. Animal Behaviour, 81, 433-438.

van de Waal, E., Renevey, N., Favre, C. M. \& Bshary, R. 2010. Selective attention to philopatric models causes directed social learning in wild vervet monkeys. Proceedings of the Royal Society B: Biological Sciences, 277, 2105-2111.

Voelkl, B. \& Noë, R. 2008. The influence of social structure on the propagation of social information in artificial primate groups: A graph-based simulation approach. Journal of Theoretical Biology, 252, 77-86.

Webster, S. J. \& Lefebvre, L. 2001. Problem solving and neophobia in a columbiformpasseriform assemblage in Barbados. Animal Behaviour, 62, 23-32.

Whiten, A. 2000. Primate culture and social learning. Cognitive Science, 24, 477-508. 


\section{Table 1 (on next page)}

Personality predicts the propensity for social learning in a wild primate Factors affecting task solving propensity (Q1), the attention given to a demonstrator (Q2), and social learning (Q3) in wild chacma baboons for two experiments (novel foo

Significance of fixed terms was determined by t- and p-values for GLMMs $(<0.05)$ and zvalues for LMEs $(\geq 2.00$ ), and random terms by a log-likelihood ratio test. The reference categories are: adult (for Age class), female (for Sex), test (for Test) and control (for Condition). 


\begin{tabular}{|c|c|c|c|c|c|c|c|c|c|}
\hline $\begin{array}{l}\text { Experimen } \\
\mathrm{t}\end{array}$ & Question & Response & $\begin{array}{l}\mathrm{N}_{\text {obs, }} \mathrm{N}_{\text {ind, }} \\
\left(\mathrm{N}_{\text {demonstrators }}\right)\end{array}$ & Deviance & Term & $\beta$ & S.E/S.D. & $\begin{array}{c}\text { test } \\
\text { statistic }^{a}\end{array}$ & $p$ \\
\hline \multirow[t]{17}{*}{ Novel food } & 2 & $\begin{array}{l}\text { Time spent } \\
\text { watching } \\
\text { demonstrator (s) }\end{array}$ & $54,36,(14)$ & 180.5 & Intercept & 2.59 & 0.52 & 5.02 & \\
\hline & & & & & Anxiety & -2.79 & 0.51 & -2.01 & \\
\hline & & & & & $\begin{array}{l}\text { Age class: } \\
\text { juvenile }\end{array}$ & 1.75 & 0.40 & 4.36 & \\
\hline & & & & & Rank & -1.74 & 0.72 & -2.43 & \\
\hline & & & & & $\begin{array}{l}\text { Random: } \\
\text { Baboon }\end{array}$ & 0.00 & 0.00 & 0.00 & 1.00 \\
\hline & & & & & $\begin{array}{l}\text { Random: } \\
\text { Troop }\end{array}$ & 1.68 & 0.51 & 0.75 & 0.39 \\
\hline & & 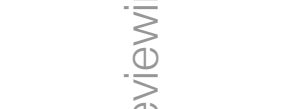 & & & $\begin{array}{l}\text { Random: } \\
\text { Demonstrator }\end{array}$ & 0.00 & 0.00 & 0.06 & 0.81 \\
\hline & $\begin{array}{l}3 \\
\text { (boldness) }\end{array}$ & $\begin{array}{l}\text { Time handling } \\
\text { food (S) }\end{array}$ & 90,32 & 1206.00 & Intercept & 1.00 & 0.47 & 2.11 & $<0.03$ \\
\hline & & 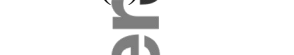 & & & Test: re-test & -0.78 & 0.08 & -9.57 & $<0.001$ \\
\hline & & (1) & & & Boldness & -1.91 & 0.51 & -3.77 & $<0.001$ \\
\hline & & Q & & & $\begin{array}{l}\text { Condition: } \\
\text { treatment }\end{array}$ & 0.17 & 0.16 & -1.09 & 0.28 \\
\hline & & & & & $\begin{array}{l}\text { Test: re-test * } \\
\text { Boldness }\end{array}$ & -0.14 & 0.05 & -2.69 & 0.007 \\
\hline & & & & & $\begin{array}{l}\text { Test: re-test * } \\
\text { Condition: } \\
\text { treatment }\end{array}$ & 0.84 & 0.10 & 8.58 & $<0.001$ \\
\hline & & & & & $\begin{array}{l}\text { Boldness * } \\
\text { Condition: } \\
\text { treatment }\end{array}$ & -0.20 & 0.25 & -0.79 & 0.43 \\
\hline & & & & & $\begin{array}{l}\text { Test: re-test * } \\
\text { Boldness * } \\
\text { Condition: } \\
\text { treatment }\end{array}$ & -0.68 & 0.08 & -8.67 & $<0.001$ \\
\hline & & & & & $\begin{array}{l}\text { Random: } \\
\text { Baboon }\end{array}$ & 5.99 & 2.45 & 3090.90 & $<0.001$ \\
\hline & & & & & $\begin{array}{l}\text { Random: } \\
\text { Troop }\end{array}$ & 0.00 & 0.00 & 0.00 & 1.00 \\
\hline
\end{tabular}


$\begin{array}{ll}3 \text { (anxiety) } & \text { Time handling } \\ & \text { food (s) }\end{array}$

90,32

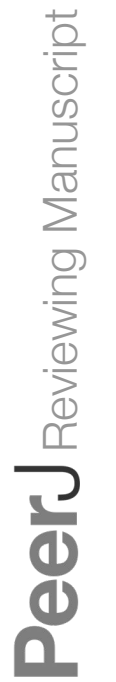

Hidden 1

food

Probability of eating food $(0 / 1)$

2

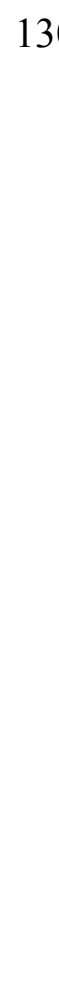

(301.00

Intercept
Test: re-test
Anxiety

1.00

0.87

1.15

0.25

Anxiety

16.11

0.09

$-9.98$

$<0.001$

Condition:

$-0.03$

5.89

2.73
-0.31

0.006

treatment

Age class:

$3.61 \quad 1.05$

juvenile

Test: re-test*

Anxiety

Test: re-test

$-11.95$

3.02

$3.43<0.001$

Condition:

treatment

Anxiety *

Condition:

treatment

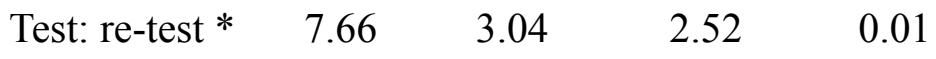

Anxiety*

Condition:

treatment

Random:

7.49

0.87

$3430.00<0.001$

Baboon

Random:

Troop

64.09 Intercept

$0.34 \quad 0.58$

0.10

0.75

Age class:

$-2.40$

1.38

$-0.74 \quad 0.08$

juvenile

Boldness

2.57

1.02

2.52

0.01

Random:

$-0.85$

0.32

$-2.63$

0.00

0.008

Baboon

Random:

Troop

Intercept

Presentation

number

Rank

.

2.03

1.43

3.85

0.05

$-0.69$

0.21

0.37

$-1.85$

2.82

0.06

$-2.0$

0.68

0.005 
Response (levels 80, 33

0-4)

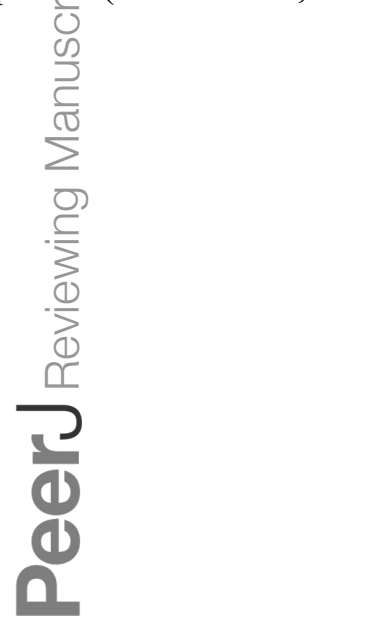

difference

Random:

Baboon

Random:

Troop

Random:

Demonstrator

208.40

Intercept

$1.04 \quad 1.02$

5.50

0.02

Boldness

Age class:

juvenile

Random:

Baboon

Random:

Troop

$\begin{array}{llll}0.00 & 0.00 & 0.00 & 1.00\end{array}$

0.06

0.24

0.06

0.80

$\begin{array}{lll}1.85 & 0.69 & 2.70\end{array}$

$-0.29$

0.10

$-2.81$

1.34

.22

6.04

0.06

0.25

7.29

0.007

0.85

0.92

0.00

1.00 


\section{Table 2 (on next page)}

The questions tested, experiments conducted, data and models used to test the predictions, and whether we found support for our predictions or not (outlined briefly here).

Predictions highlighted in bold show that we found support for these in our models. Note that this may indicate that there was not a significant relationship. Predictions for which we did not find support but found the opposite trend are in bold and italicized. 'N/A' is listed for both boldness and anxiety effects in the novel food experiment under Question 1. This is because, in the case of boldness, this experiment was also our assay for boldness, and in the case of anxiety, previous work has demonstrated that anxiety is unrelated to boldness (and hence performance in the novel food experiment) in this species (Carter et al. 2012c). 
Prediction and whether it was supported

\begin{tabular}{|c|c|c|c|c|c|c|}
\hline Question & Test & Experiment & Data analysed & Model & Anxiety & Boldness \\
\hline \multirow[t]{4}{*}{1} & \multirow{4}{*}{$\begin{array}{l}\text { Propensity to } \\
\text { solve the task }\end{array}$} & $\frac{\sqrt{\sigma}}{\bar{c}} \mathrm{vel}$ food & $\mathrm{N} / \mathrm{A}$ & & N/A & N/A \\
\hline & & Hiddden food & $\begin{array}{l}\text { Whether the baboon ate } \\
\text { food from the task or }\end{array}$ & $\mathrm{m} 1$ & $\begin{array}{l}\text { P1a: calm-anxious } \\
\text { animals unlikely to }\end{array}$ & $\begin{array}{l}\text { P1b: bold animals are } \\
\text { likely to solve }\end{array}$ \\
\hline & & $\geq$ & $\operatorname{not}(0 / 1)$ & & differ & YES \\
\hline & & & & & YES & \\
\hline \multirow[t]{4}{*}{2} & \multirow[t]{4}{*}{$\begin{array}{l}\text { Watching a } \\
\text { demonstrator }\end{array}$} & $\begin{array}{l}\text { Novel food } \\
\simeq\end{array}$ & \multirow[t]{2}{*}{$\begin{array}{l}\text { Time (s) spent watching } \\
\text { a demonstrator }\end{array}$} & \multirow[t]{2}{*}{$\mathrm{m} 2 \mathrm{nf}$} & $\begin{array}{l}\text { P2a: anxious animals } \\
\text { will be more attentive }\end{array}$ & $\begin{array}{l}\text { P2b: shy animals will } \\
\text { be more attentive }\end{array}$ \\
\hline & & 3 & & & $\begin{array}{l}\text { No, calmer individuals } \\
\text { were more attentive }\end{array}$ & NO (neither) \\
\hline & & 1idden food & \multirow{2}{*}{$\begin{array}{l}\text { Whether the baboon } \\
\text { watched a demonstrator } \\
\text { or not }(0 / 1)\end{array}$} & \multirow[t]{2}{*}{$\mathrm{m} 2 \mathrm{hf}$} & $\begin{array}{l}\text { P2a: anxious animals } \\
\text { will be more attentive }\end{array}$ & $\begin{array}{l}\text { P2b: shy animals will } \\
\text { be more attentive }\end{array}$ \\
\hline & & & & & No (neither) & NO (neither) \\
\hline \multirow[t]{4}{*}{3} & \multirow{4}{*}{$\begin{array}{l}\text { Change in task- } \\
\text { solving } \\
\text { behaviour after } \\
\text { watching a } \\
\text { demonstrator }\end{array}$} & Novel food & \multirow[t]{2}{*}{$\begin{array}{l}\text { Time spent handling the } \\
\text { novel food (s) after } \\
\text { treatment/control }\end{array}$} & \multirow[t]{2}{*}{$\mathrm{m} 3 \mathrm{nf}$} & $\begin{array}{l}\text { P3a: anxious animals } \\
\text { will show greater } \\
\text { improvement }\end{array}$ & $\begin{array}{l}\text { P3b: shy animals will } \\
\text { show greater } \\
\text { improvement }\end{array}$ \\
\hline & & & & & YES & $\begin{array}{l}\text { NO, bold individuals } \\
\text { improved. }\end{array}$ \\
\hline & & \multirow[t]{2}{*}{ Hidden food } & \multirow[t]{2}{*}{$\begin{array}{l}\text { Level of interaction } \\
\text { with the hidden food } \\
\text { container (levels } 0-4 \text { ) }\end{array}$} & \multirow[t]{2}{*}{$\begin{array}{l}\text { Model set } \\
\text { 3hf }\end{array}$} & $\begin{array}{l}\text { P3a: anxious animals } \\
\text { will show greater } \\
\text { improvement }\end{array}$ & $\begin{array}{l}\text { P3b: shy animals will } \\
\text { show greater } \\
\text { improvement }\end{array}$ \\
\hline & & & & & NO (neither) & NO (neither) \\
\hline
\end{tabular}




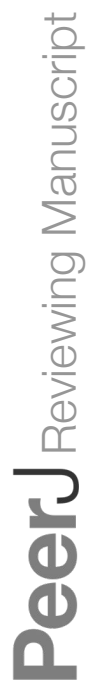




\section{Figure 1}

Personality predicts the propensity for social learning in a wild primate The times that individuals spent watching a demonstrator manipulate a novel food

The average time anxious and calm juvenile and adult baboons spent watching a demonstrator (s) interact with a novel food ( $n=54$ trials). Plotted are the means and standard errors for the raw data; note that though the data are presented as categories (split at the mean of the trait for the population) they were analysed as continuous variables. 


\section{Figure 2}

Personality predicts the propensity for social learning in a wild primate Individual responses to a novel food after an opportunity for social learning

The average time that bold and shy (a, b) and anxious and calm (c, d) baboons handled the novel food on their first interaction with it (pres. 1) and on their second interaction with it (pres. 2) either without being exposed to a demonstrator (a, c) or after being exposed to a demonstrator $(b, d)(n=45$ trials). Plotted are the means and standard errors for the raw data; note that though the data are presented as categories (split at the mean of the trait for the population) they were analysed as continuous variables. 
Control
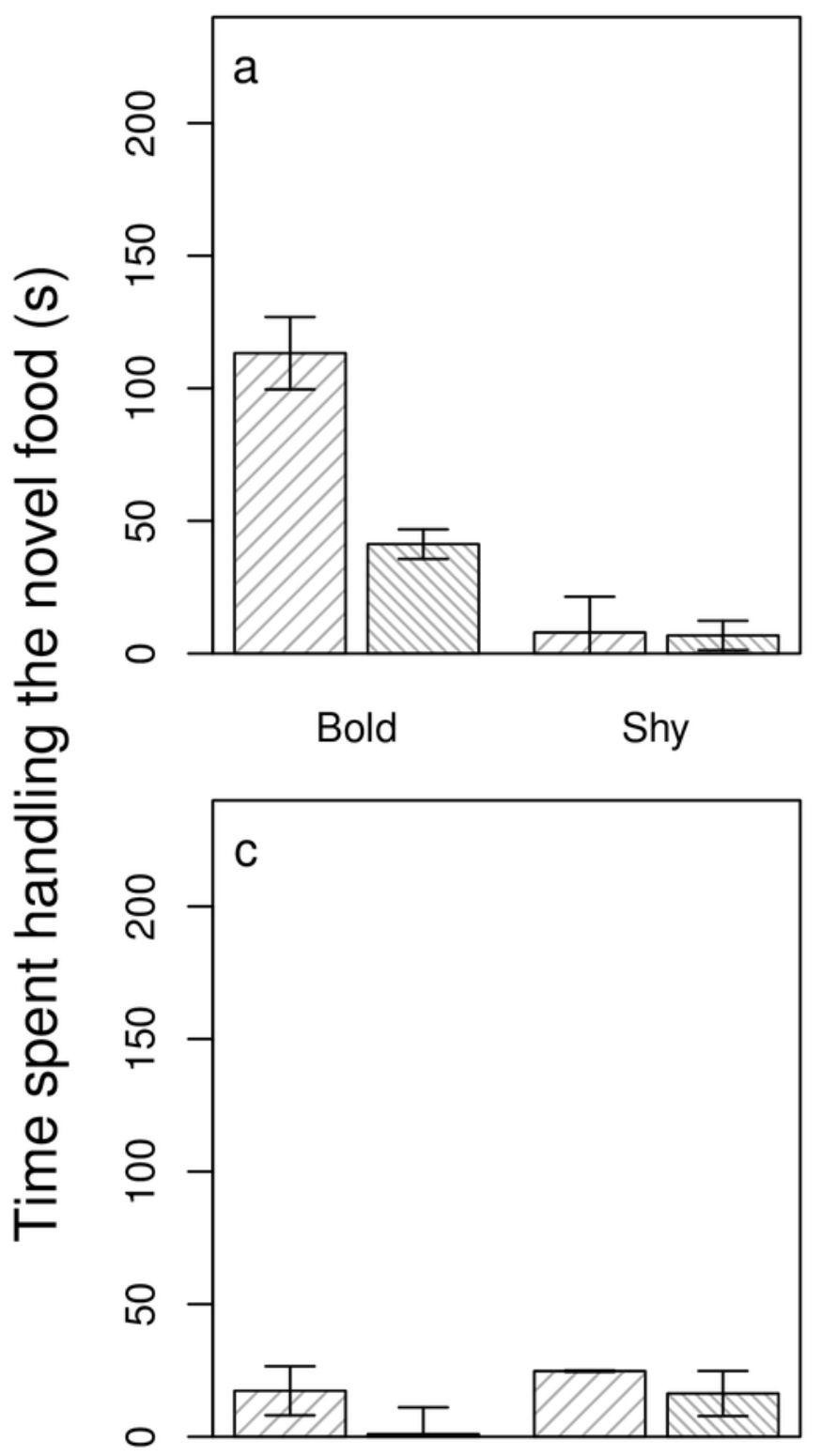

Anxious
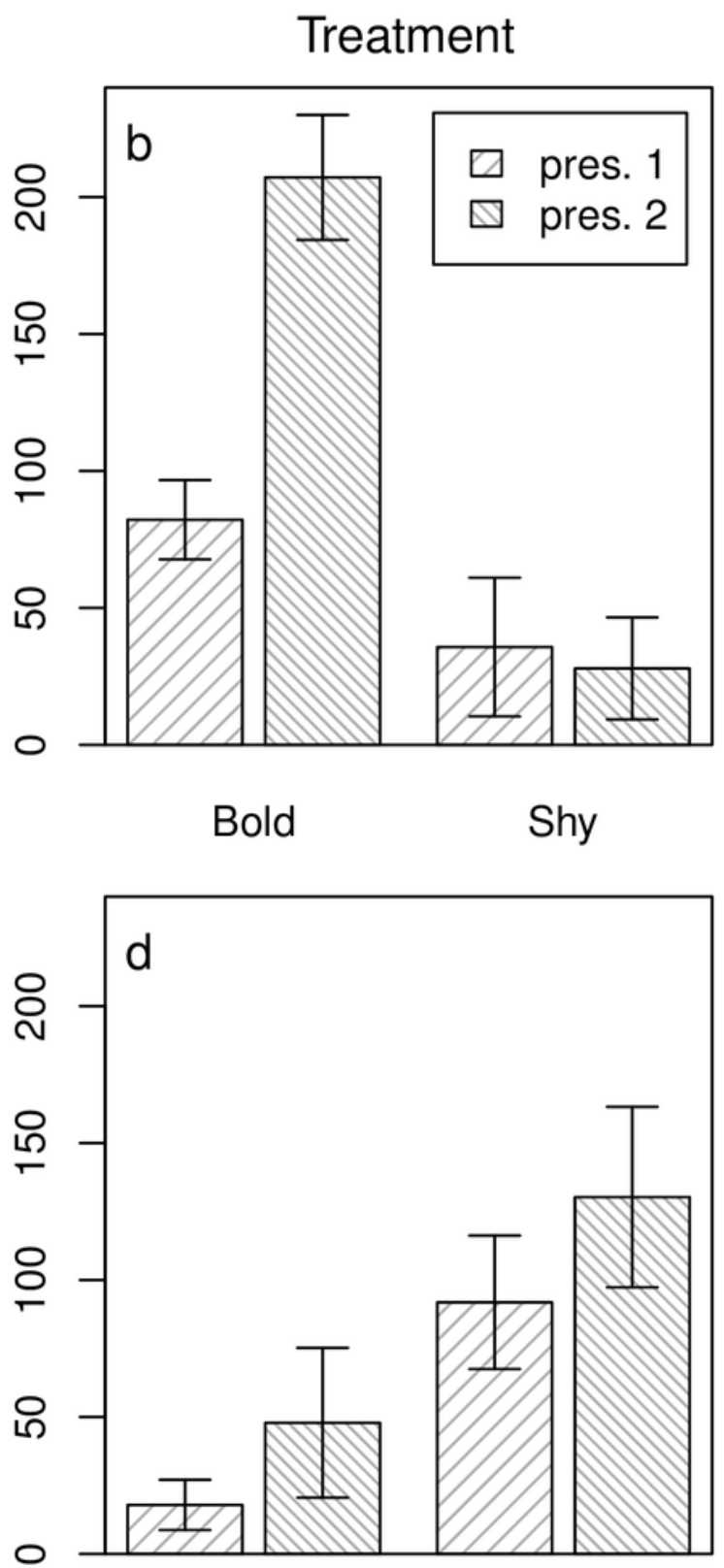

Anxious

Calm 


\section{Figure 3}

Personality predicts the propensity for social learning in a wild primate The proportions of individuals that solved the task

Differences in proportions of adult and juvenile bold and shy baboons that 'solved' the hidden food task by obtaining and eating food from the box $(n=80)$. Plotted are the means and standard errors for the raw data; note that though the data are presented as categories (split at the mean of the trait for the population) they were analysed as continuous variables. 
